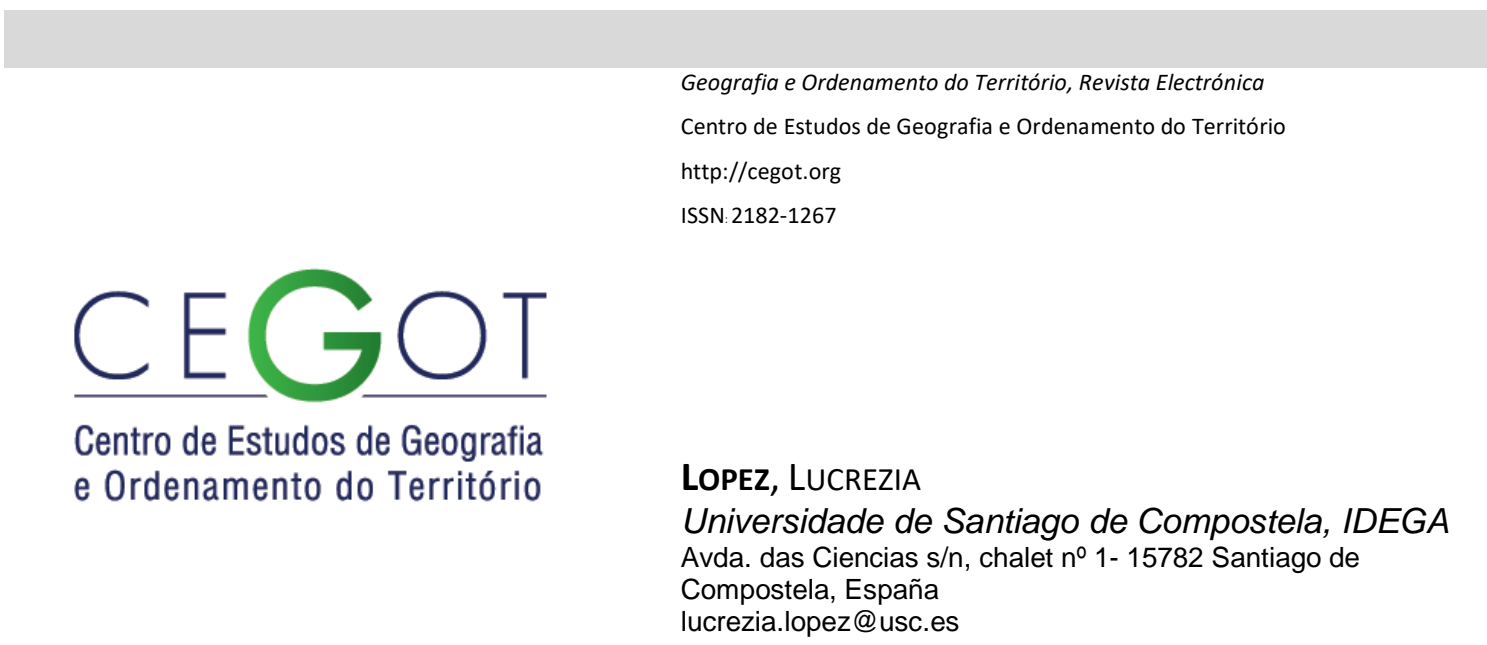

\title{
Nuevas fórmulas para un desarrollo local sustentable. Los Ecomuseos en Apulia (Italia)
}

\author{
Novas fórmulas para um desenvolvimento local sustentável. Os Ecomuseus \\ em Apúlia (Itália) \\ New formulas for sustainable local development. The Ecomuseums in Apulia \\ (Italy)
}

Referência: Lopez, Lucrezia (2017). Nuevas fórmulas para un desarrollo local sustentable. Los Ecomuseos en Apulia (Italia). Revista de Geografia e Ordenamento do Território (GOT), n.o 11 (junho). Centro de Estudos de Geografia e Ordenamento do Território, p. 241-262, dx.doi.org/10.17127/got/2017.11.011

\section{RESUMEN}

Con la Ley Regional del 6 de Julio 2011, el Consejo Regional de la Pulla (Italia) fija la institución de los ecomuseos en la misma región. Tal como se recoge, los objetivos son promover y regular los ecomuseos para recuperar y mejorar la memoria histórica, la vida, la cultura tangible e intangible, las relaciones entre el medio ambiente, las tradiciones naturales y artificiales, las actividades y las tradiciones del paisaje y de la región. Se analizan los ecomuseos de la región costera italiana (contenidos de los proyectos, actores, objetivos, actuaciones y herramientas). Se investiga de qué manera están contribuyendo a la promoción del patrimonio cultural y de la dimensión local y social. Se procede reconstruyendo su metodología de trabajo y se avanza un balance de las herramientas y de las modalidades de conocimiento y valorización del patrimonio en relación al desarrollo local y sustentable.

Palabras-clave: Ecomuseos, Apulia (Italia), Desarrollo Local Sustentable, Mapa de Comunidad. 


\title{
RESUMO
}

Com a Lei Regional de 6 de julho de 2011, o Conselho Regional de Pulla (Itália) determina o estabelecimento de ecomuseus nesta região. Tal como se mostra, os objetivos são promover e regulamentar os Ecomuseus para recuperar e melhorar a memória histórica, a vida, a cultura material e imaterial, as relações entre meio ambiente, tradições naturais e artificiais, atividades e tradições da paisagem e da região. Analisam-se os Ecomuseus da região costeira italiana (conteúdo dos projetos, atores, objetivos, ações e ferramentas). Adicionalmente, investiga-se de que forma estes estão a contribuir para a promoção do património cultural e da dimensão local e social. Por fim procede-se à reconstituição da metodologia de trabalho e realiza-se um balanço das ferramentas e modos de conhecimento e valorização do património em relação ao desenvolvimento local e sustentável.

Palavras-chave: Ecomuseus, Apúlia (Itália), Desenvolvimento local sustentável, mapa da Comunidade.

\begin{abstract}
With the Regional Law of 6 July 2011, the Regional Council of Apulia (Italy) establishes the institution of the ecomuseums in the same region. The objectives are to promote and regulate ecomuseums in order to recover and improve historical memory, life, tangible and intangible culture, relationships between the environment, natural and artificial traditions, activities and landscape and regional traditions. The contribution presents the analysis of the ecomuseums of the Italian coastal region (contents of projects, actors, objectives, actions, tools, etc.). It investigates how they are contributing to the promotion of cultural heritage and to the local and social dimension. After presenting the methodology, the essay presents a balance of the tools and methods of knowledge and valorization of the heritage in relation to local and sustainable development.
\end{abstract}

Key-words: Ecomuseums, Apulia (Italy), Sustainable Local Development, Community Map.

\section{Introducción}

La idea original del Ecomuseo nace durante una reunión internacional del ICOM en el 1972, a la vez que se elabora una primera definición "oficial" del mismo, redactada por Georges Henri Rivière: "un ecomuseo es un instrumento ideado, fabricado y explotado conjuntamente por un poder y una población, entendiendo por poder los expertos, las facilidades y los recursos que éste aporta, y por población las aspiraciones, los conocimientos y las facultades de aproximación de la misma (...)" (citado por DE VARINE, 2007: 28). Ya en esta definición se hace alusión a la naturaleza participada de los ecomuseos, que queda hoy 
reflejada en una práctica común: el mapa de comunidad (CARTA, 2011). Introducido en Inglaterra a comienzo de los años 80 , en su representación colabora la visión local de su patrimonio y de las relaciones territoriales. Derivan de un proceso comunicativo y participativo entre habitantes que, reuniendo saberes y conocimientos, representan el territorio desde un punto de vista cualitativo. Un mapa debe ser construido de una manera participativa, ya que permite a la comunidad afectada reconocer el valor de los lugares y aumentar su autoestima. En definitiva, es un método de crecimiento colectivo y autoformación para colectivos locales, formados por jóvenes, a menudo indiferentes al contexto de pertenencia. El resultado es un espacio vivido que expresa los valores socialmente reconocidos en el territorio.

Por su parte, el museólogo Hugues de Varine replantea el concepto de museo como ecomuseo, la colección como patrimonio, el contenedor como territorio y el público como comunidad. Según cuanto definido en la Carta de los Ecomuseos: "El Ecomuseo es una institución cultural que asegura permanentemente, en un territorio dado y con la participación ciudadana, las funciones de investigación, conservación y puesta en valor de un conjunto de bienes naturales y culturales representativos de un entorno y de las formas de vida que han tenido lugar".

La importancia de la percepción del territorio responde a las indicaciones del Convenio Europeo del Paisaje, presentado en Florencia el 20 octubre del año 2000 (BARATTI, 2012). En el preámbulo se indica que: "el paisaje contribuye a la formación de las culturas locales y que es un componente fundamental del patrimonio natural y cultural europeo, que contribuye al bienestar de los seres humanos y a la consolidación de la identidad Europea". Según el Consejo de Europa: "por "paisaje" se entenderá cualquier parte del territorio tal como la percibe la población, cuyo carácter sea el resultado de la acción y la interacción de factores naturales y/o humanos" (Artículo 2). El documento europeo reconoce a los paisajes europeos el valor de patrimonio común, pilares de la identidad europea. A raíz de los valores del paisaje, las finalidades del Convenio son: "promover la protección, gestión y ordenación de los paisajes, así como organizar la cooperación europea en ese campo" (Artículo 3). Según el Convenio Europeo del Paisaje, la protección del paisaje implica todas las acciones para conservar y mantener el significado y las características típicas del mismo, 
además de crear un repertorio para definir las condiciones del sistema de gestión del paisaje.

\subsection{Metodología}

El presente estudio se estructura en cinco apartados principales, además de la introducción y de las conclusiones. En primer lugar, se presentan algunas de las definiciones teóricas más relevantes en relación a los ecomuseos y a sus factores de éxito. Esta aproximación queda reflejada en la figura 1, en la cual se elabora un esquema interpretativo de los pilares de los ecomuseos para el desarrollo rural sustentable. Se procede a analizar el caso de la región italiana de Apulia. En Italia, la experiencia empieza en la región de Piamonte (L. R. 31/95), siguen la Provincia Autónoma de Trento y la región Autónoma de Friuli Venecia Julia (L. R. n. 10 del 20.06.2006). En la Región de Apulia, esta fórmula queda instituida con la Ley Regional del 6 de Julio 2011, n. 15, cuyo artículo 1 define los objetivos de los ecomuseos: "recuperar, presenciar, mejorar y acompañar en su desarrollo la memoria histórica, la vida, la figuras y los hechos, la cultura tangible e intangible, las relaciones entre el entorno natural y el medio ambiente antropizado, las tradiciones, las actividades y la manera en que el establecimiento tradicional ha caracterizado a la formación y a la evolución del paisaje y de la región, para orientar el desarrollo futuro del territorio según una lógica de sostenibilidad medioambiental, económica y social, de responsabilidades y de participación de sujetos públicos y privados en la comunicad local".

Se opta por una metodología cualitativa basada en la recopilación de los principales proyectos puestos en marcha en la región en términos de participación ciudadana y promoción de actividades. Tras esta selección, se analizan las experiencias en función de las siguientes variables: metodología de trabajo, actores, objetivos, actuaciones y herramientas. A través de este enfoque analítico se quiere averiguar de qué manera están contribuyendo a la promoción del patrimonio cultural y de la dimensión local y social, como reflejado en el punto el análisis de las potencialidades de los Ecomuseos para un desarrollo rural sostenible. 
Retomando la definición ya citada de Georges Henri Rivière y a partir de los casos de estudio, se pone de relieve el uso que los ecomuseos están haciendo de una técnica de planificación participada: el mapa de comunidad. Así mismo, esta contribución se convierte en una ocasión para conocer el proceso de construcción de los mapas de comunidad, instrumentos de producción social del paisaje, en el marco del nuevo Plan Paisajístico Territorial Regional (PPTR) en Apulia. A través de este caso regional, se quiere compartir la utilidad de emplear, en el marco de los ecomuseos, la técnica de los mapas de comunidad como herramienta de conocimiento, comunicación y planificación del paisaje.

\section{2. ¿Qué es un Ecomuseo? Algunas reflexiones teóricas}

La principal definición de Ecomuseo ha sido formulada por Henri Rivière, según el cual se trata de: "Un espejo donde la población se contempla para reconocerse, donde busca una explicación del territorio en el que está enraizada y en el que se sucedieron todos los pueblos que la precedieron en la continuidad o discontinuidad de las generaciones. Un espejo que la población ofrece a sus huéspedes para hacerse entender mejor, en el respeto de su trabajo, de sus formas de comportamiento y de su identidad. Una expresión del hombre y de la naturaleza. El hombre es allí interpretado en su relación a su ámbito natural y la naturaleza está presente en su estado salvaje, pero también tal como la sociedad tradicional y la sociedad industrial la transforman a su imagen" (RIVIÈRE, 1985: 182).

Inicialmente, el ecomuseo surge como un museo dedicado al medioambiente, con un fuerte componente de identificación con el territorio y con la población del mismo: "Se trata de una estructura de lenguaje expositivo que cubre la totalidad de un territorio más o menos coherente y que utiliza el patrimonio global de dicho territorio, así como las habilidades de sus habitantes. Es un instrumento cultural para el desarrollo del territorio, según un proceso continuo que involucra a la comunidad local, a los colectivos políticos y a las instituciones administrativas y científicas, al igual que a un cierto número de profesionales (DE VARINE, 2007: 22). Asimismo, se entiende el ecomuseo como parte de instrumentos de la dimensión cultural del desarrollo local; cumple con otros objetivos en común con los museos ordinarios, es decir, el desarrollo de la cultura, la conservación del patrimonio, la acogida de turistas, la formación de alumnos, etc. 
Otra definición fue elaborada por Ewa Bergdahl (1998), según la cual el ecomuseo es un instrumento dedicado al desarrollo regional en cuyo proceso se pone en colaboración la población local con las instituciones públicas. Efectivamente, ese es uno de los factores positivos del ecomuseo, que a partir de sus distintas técnicas de realización, fomenta el diálogo y la escucha entre la visión ciudadana y la administrativa. Como se verá más adelante, ese ha sido el caso de los ecomuseos de Apulia, que gracias a los mapas de comunidad han establecido una forma de comunicación bottom-up. Según Ignacio Muñiz (2007), el ecomuseo se materializa a partir de diferentes núcleos museísticos e infraestructuras dispersas por el término municipal, teniendo muy en cuenta la participación de la población en cuanto que es parte de ese patrimonio.

Para los ecomuseos, los elementos más importantes son los conocimientos, los saberes y las temáticas de reflexión que puede generar el patrimonio (MORIL, 2009). De ahí, que su objetivo principal es transmitir información. En general, de la misma manera que las otras tipologías de museos, las funciones que debe de cumplir un ecomuseo son: 1) Documentación; 2) Difusión y comunicación; 3) Conservación; 4) Investigación (BELTRÁN, 2012). El éxito de un ecomuseo reside en la participación ciudadana, ya que de ello depende el hecho de convertirse en motor del desarrollo local. Por esta razón, los ecomuseos deben ser creados como algo dinámico, accesible y apto para todos los públicos con independencia de la edad y el nivel cultural (BELTRÁN, 2012). Sin embargo, uno de los riesgos que corre el ecomuseo es que acabe siendo un parque temático, en el cual el patrimonio ya no lleve a la reflexión.

A partir de esas aproximaciones teóricas, se ha elaborado la figura 1 en la cual se resumen los pilares del ecomuseo, para que este tenga como fin un desarrollo endógeno y equilibrado de su localidad (FERNÁNDEZ, 2004). La población y su participación son fundamentales para recuperar y dar a conocer la historia del territorio, conservar e interpretar el patrimonio. Es ese caso, se entiende la palabra patrimonio como el conjunto de elementos materiales e inmateriales, así que se incluye la herencia del pasado, los saberes, y los elementos patrimoniales materiales. El ecomuseo, como otras categorías museológicas, posee una función didáctica para dar a conocer, comunicar y hacer 
comprensibles los bienes patrimoniales a un público general o específico (SANTACANA y SERRAT, 2007).

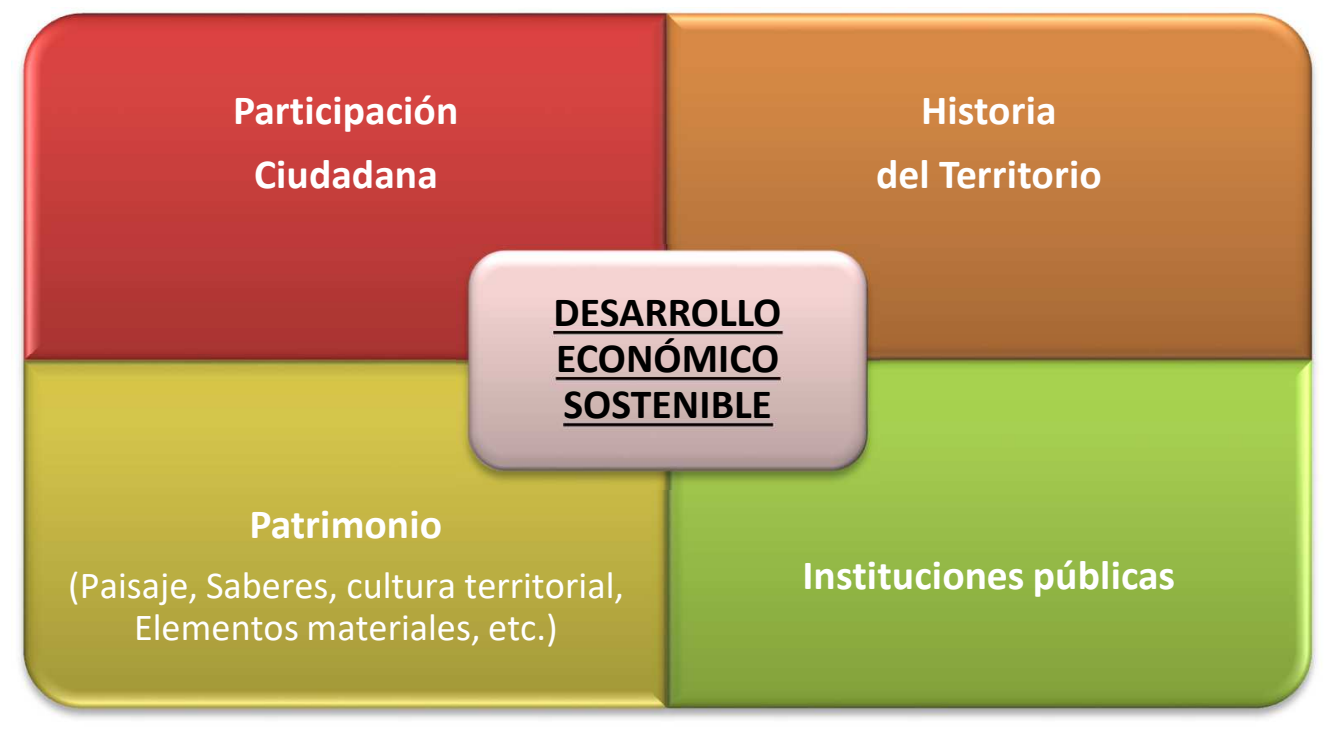

Figura 1 - Los Pilares del Desarrollo Económico Sostenible a través de un Ecomuseo. Fuente: Elaboración Propia.

En la Península Ibérica el ecomuseo penetra por Cataluña, con establecimientos como el de las minas prehistóricas de Gavá o el ecomuseo de Les Valls d’Àneu, en los Pirineos, uno de los pioneros (1994) en implantar una nueva visión de la gestión territorial de los recursos patrimoniales (FERNÁNDEZ, 2004; BELTRÁN, 2012). Otro ejemplo relevante, también premiado para su labor, es el ecomuseo del Río Caicena en Almedinilla (Córdoba). Su trabajo se centra en realizar actividades de divulgación. En esta población, en los años previos a la instalación del ecomuseo se habían dado una progresiva pérdida de identidad cultural y una masiva emigración a la capital, con lo que el futuro de la población estaba condenado. Un plan de desarrollo basado en la puesta en valor de los bienes culturales ha supuesto una creación de empleo y de pequeñas empresas familiares en torno al proyecto, que han conseguido evitar la situación que se estaba produciendo hasta ese momento. La CC. AA. de Galicia cuenta, entre otros, con el ecomuseo "Terras de Azul Cobalto"; un proyecto de musealización de todos los recursos naturales, tradicionales, patrimoniales y turísticos del territorio municipal de O Cervo. Su objetivo es convertirse en seña de identidad mostrando la riqueza paisajística, histórica y humana. O el ecomuseo de Arxeriz, que forma parte de la 
Red Museística de Galicia desde el 28 de Noviembre 2008. Nacido en el 2004, se plantea amparar, rescatar, defender, restaurar, estudiar e investigar al patrimonio etnográfico e histórico-artístico de la Ribera Sacra. En general, en España se está asistiendo a la difusión de esos proyectos de desarrollo territorial a partir del patrimonio histórico-natural; todos comparten objetivos similares, aunque se está trabajando en lo referente a las prácticas de participación ciudadana.

\section{Los Ecomuseos en Apulia}

El arranque de los Ecomuseos en Apulia ha fomentado el uso de nuevas herramientas para la puesta en valor del patrimonio paisajístico y el desarrollo local y sostenible. En el marco del Plan Paisajístico Territorial Regional (PPTR), el Ecomuseo contribuye a la aparición de nuevos significados y valores del paisaje, sensibilizando las poblaciones hacia sus raíces históricas y culturales. De ahí que el PPTR de Apulia es un ejemplo de planificación que transmite los valores territoriales e identitarios (CARTA, 2011).

Según cuanto indicado en la Resolución Regional del 29 Enero 2015, n. 48, en Apulia existen nueve ecomuseos, en los cuales se lleva a cabo el proyecto experimental del mapa de Comunidad. Para hacer frente a la gestión de los ecomuseos, se ha instituido la Rete Ecomusei Puglia; a esa red participan todos los ecomuseos regionales, que cooperan en la gestión y en la organización de los sistemas ya existentes (BARATTI, 2012).

\subsection{El Ecomuseo de Valle d'Itria}

En Mayo 2009 los ayuntamientos de Alberobello, Cisternino, Fasano, Locorotondo, Martina Franca y Monopoli firmaron un memorando de entendimiento para constituir el Ecomuseo della Valle d'Itria. El jefe de fila del proyecto fue el alcalde de Locorotondo, encargado de programar y establecer intenciones en relación a la constitución del ecomuseo. Sucesivamente, los ayuntamientos de Alberobello, Cisternino, Fasano, Locorotondo, Martina Franca y Monopoli firmaron un memorando de entendimiento con la Región Apulia 
para constituir el Ecomuseo de Valle d'Itria, proyecto piloto en el marco del PPTR. El 13 de Febrero del 2010 fue instituida la Asociación Cultural, sin ánimo de lucro, denominada: "Asociación de Ecomuseos de Valle d'Itria" para la gestión del ecomuseo. La misma sigue funcionando a través de iniciativas destinadas a las comunidades, promoviendo la colaboración con asociaciones y grupos de personas que trabajan en el territorio, con la firme intención de ser un museo difuso del territorio. El trabajo está coordinado por el Laboratorio di Urbanística Partecipata. El valor añadido del proyecto es fomentar un desarrollo basado en la participación activa de ciudadanía joven para reconstruir la identidad de los lugares habitados.

El museo recoge distintos elementos naturales y antrópicos que se vinculan a la historia geológica y al uso del territorio. Este museo del territorio persigue objetivos comunes a los de un museo, es decir, documentar, guardar y valorizar elementos materiales e inmateriales, que en este caso son sitios naturales y manifestaciones de la cultura. Por otro lado, se plantea objetivos más específicos cuales:

- Favorecer la integración y la participación activa de jóvenes en la vida de la comunidad;

- Apoyar redes territoriales de agregación juvenil finalizadas al redescubrimiento de la identidad local;

- Reforzar la capacidad juvenil de asumir responsabilidades y trabajar autónomamente;

- Favorecer una aproximación positiva al territorio por parte de jóvenes generaciones.

Las características estructurales de este "museo del territorio" son:

- Presencia de una compleja herencia local profundamente ligada al territorio que lo identifica (genius loci);

- Enfoque interdisciplinar y holístico de las actividades de valorización;

- Atención a la relación entre comunidad y territorio y a su desarrollo (recuperación de las raíces históricas de la comunidad y de su memoria);

- Atención al medioambiente para mantenerlo con acciones intencionales;

- Fruición del Ecomuseo por parte de comunidades locales, principales destinatarias de las actividades programadas. 


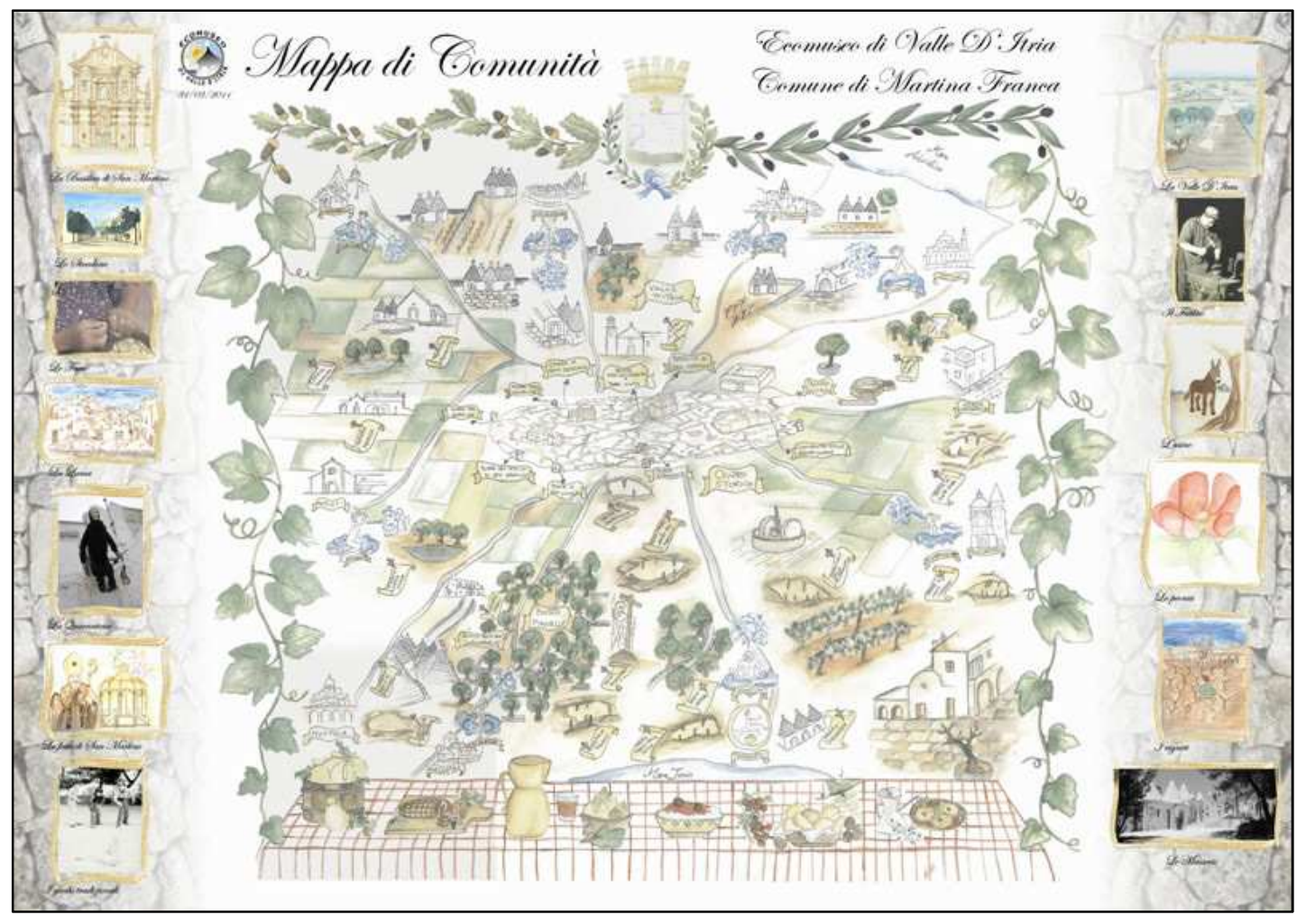

Figura 2 - Mapa de Comunidad de Martina Franca Fuente: Ecomuseo de Valle d'Itria

Desde los actores hasta los objetivos, el proyecto del ecomuseo está enfocado a la participación y fruición ciudadana, como también confirman los servicios puestos en marcha para promover la comprensión y el disfrute del patrimonio territorial: museos ciudadanos (centros de documentación abiertos al público); pluralidad de sitios para visitas y presencia de caminos o senderos temáticos que definen una red compleja de interrelaciones. También preserva su naturaleza de institución de educación mediante la puesta en marcha de actividades de formación, didáctica y educación medioambiental (laboratorio, visitas, excursiones, cursos de formación y especialización, encuentros y debates) y se representan los valores territoriales de forma innovadora. 


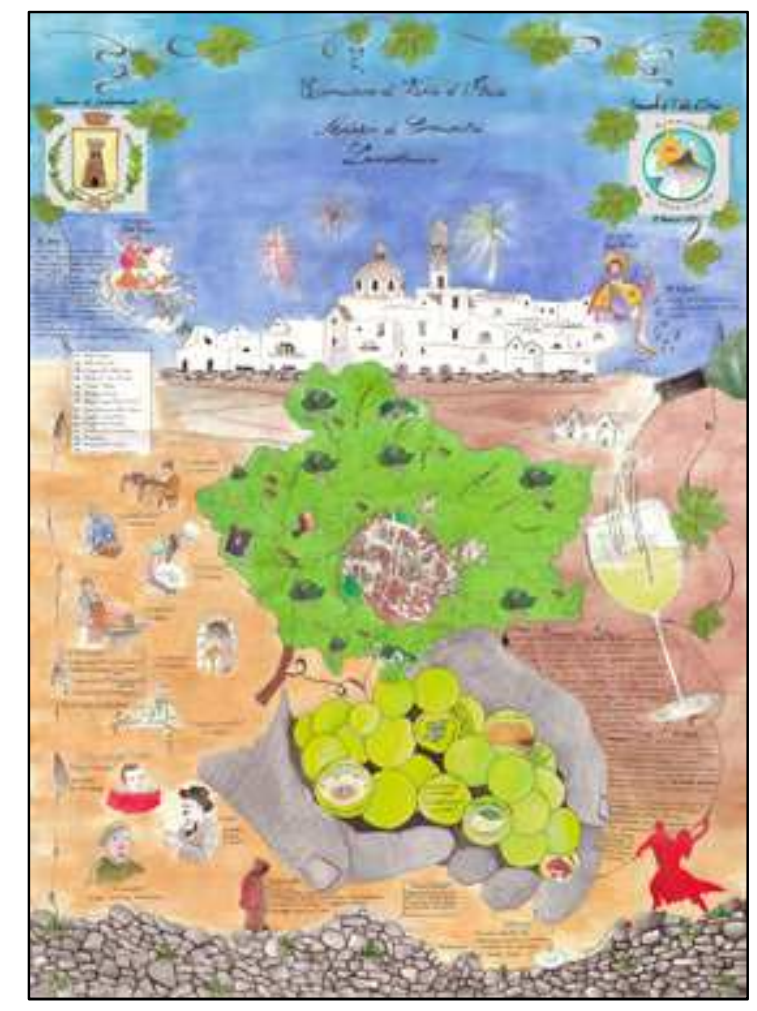

Figura 3 - Mapa de Comunidad de Locorotondo. Fuente: Ecomuseo de Valle d'Itria

Algunas de las iniciativas de sensibilización ciudadana contemplan un pequeño grupo de trabajo formado por jóvenes licenciados o con trabajos precarios, para formarlos para trabajar en el Ecomuseo, y la puesta en marcha de "laboratorios participativos", para elaborar mapas de comunidad. El resultado ha sido la activación de un mecanismo de participación para producir una dinámica fundada en la memoria. Gracias al proyecto "Mapa de Comunidad" se han destacado los rasgos territoriales. La realización del "mapa de comunidad" se ha estructurado en 7 fases:

1. Formación de un grupo de expertos para investigar metodologías de puesta en valor del patrimonio cultural, medioambiental y natural.

2. Individuación de las áreas en Valle d'Itria destinadas a ser representadas en los "mapas de comunidad". Han sido señaladas por parte de los jóvenes; presentan un carácter de homogeneidad desde el punto de vista geográfico.

3. Los jóvenes facilitadores han desempeñado su labor en grupos de trabajo. Han realizado investigaciones junto a la acción de intervención sobre el terreno. 
4. La tarea de mapeo se ha hecho mediante reuniones y seminarios; folletos y boletines de noticias, artículos e informes en la prensa local. Los jóvenes facilitadores han activado colaboraciones entre actores locales para poner en marcha una red territorial de éxito. Se ha dado importancia y atención a los conocimientos de cada habitante en relación al área de representación.

5. En una reunión pública se ha ampliado la activación a los grupos de actores locales involucrados en el proceso de realización de los mapas de comunidad.

6. Procesamiento de mapas con características físicas y biológicas, historias y saberes, lugares y personas. La representación se enriquece de fotografías, dibujos, textos y reconstrucciones.

7. Presentación en un evento público final.

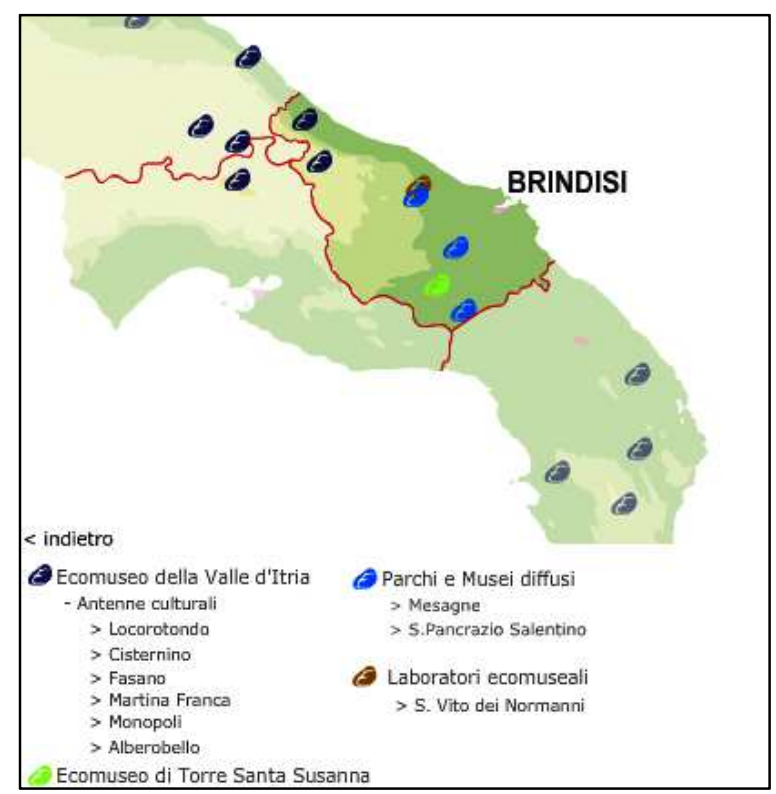

Figura 4 - Ecomuseos en la Provincia de Brindisi. Fuente: Sistema Ecomuseale della Puglia.

\subsection{Ecomuseo del Paesaggio delle Serre Salentine (Ecomuseo del Paisaje de las Sierras} de Salento)

El objetivo del Ecomuseo del Paesaggio delle Serre di Neviano ha sido poner en valor todo el territorio municipal, identificando en las Sierras (últimas prolongaciones delle Murge del Salento), que representa su identidad. Nace en el 2007 en ocasión de la recuperación de la 
abadía de San Nicola di Macugno, su restauración ${ }^{1}$ y su ubicación han sido los propulsores del laboratorio para el mapa de comunidad. Los participantes al laboratorio han cooperado para encontrar signos ocultos de la historia, la cultura, el uso de la tierra y otras acciones colectivas heredadas por ese territorio.

La lectura participada del paisaje y la construcción del mapa de comunidad de Neviano ha sido un "proyecto piloto" y puede ser considerado un ejemplo de buena práctica en Apulia. Ha tenido una conclusión exitosa ya que su población ha sido capaz de entender su papel de interlocutor privilegiado, repositorio de conocimientos y experiencias importantes. A lo largo de estos años la iniciativa se ha mantenido en pie, y como resultado la Asociación de Ecomuseo de las Sierras Salentine de Neviano está formada hoy por cuarenta personas que trabajan en actividades distintas (BARATTI, 2012). El Ecomuseo de Neviano es una realidad consolidada que incluye bienes paisajísticos y arquitectónicos. Su objetivo es dar a conocer, resanar y recuperar los elementos medioambientales e históricos del territorio. Precisamente este estudio del territorio, les ha empujado a desempeñar actividades de investigación, catalogación y estudio con el fin de señalar y salvaguardar un legado en riesgo de abandono. El proceso de recalificación de esas áreas contempla también un uso lúdico y turístico del territorio (BARATTI, 2012). En esa tarea, trabaja con los centros educativos locales en proyectos de educación ambiental, alienta a los pequeños agricultores, organiza exposiciones temáticas históricas, artísticas y fotográficas, promueve escritos por autores locales, organiza eventos de teatro en la lengua vernácula, recupera y restaura viejas tradiciones.

\footnotetext{
${ }^{1}$ Financiada por un los fondos Fondi PIS 14 - POR/Puglia y una financiación del CUIS - Consorcio Interuniversitario Salentino.
} 


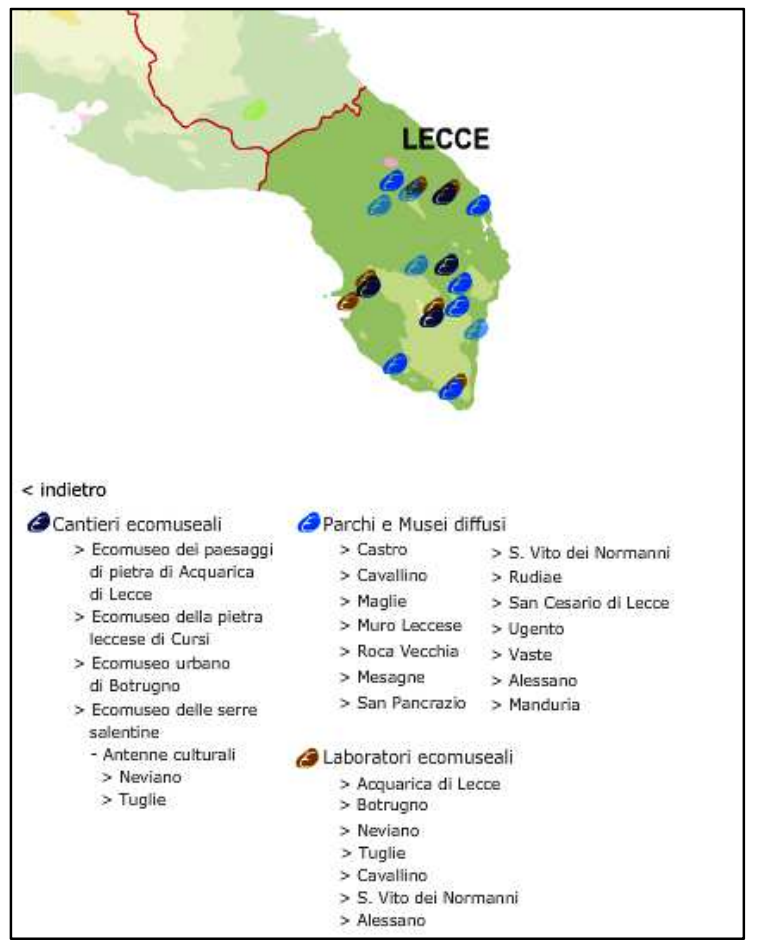

Figura 5 - Ecomuseos del Paesaggio delle Serre Salentine.

Fuente: Sistema Ecomuseale della Puglia.

\subsection{Ecomuseo della Valle del Carapelle (Ecomuseo de la Valle del río Carapelle)}

El 28 de noviembre de 2008 se lleva a cabo el primer paso del Ecomuseo con la firma de un memorando de entendimiento entre la región de Apulia, la Universidad de Foggia y los alcaldes de seis municipios: Ascoli Satriano, Ordona, Ortanova, Carapelle, Stornarella y Stornara. Todos ellos acomunados por el hecho de pertenecer al valle del río Carapelle, que durante siglos fue camino de la travesía de este territorio privilegiado que dio forma al paisaje.

En el 2012 se presenta formalmente el proyecto Ecomuseo del Valle del Carapelle, instituido como una ruta compartida del saber. En la misma línea que los demás ecomuseos, sus objetivos son recoger, preservar y promover el patrimonio cultural, las tradiciones, los objetos y proteger los paisajes en los que los habitantes se identifican. El Ecomuseo ocupa los sitios de excavación de Herdonia y Faragola, y está fuertemente comprometido con este territorio promoviendo actividades de investigación en la valorización de este patrimonio, organizando seminarios y actividades de divulgación y de promoción local. 
Los mapas comunitarios realizados reúnen historia y tradiciones de los municipios participantes. Los bienes representados por los ciudadanos, además de los ya identificados en el proyecto Carta de los Bienes Culturales de Apulia, forman una red de bienes, cuyo uso está facilitado por la presencia de centros de documentación. Hay que destacar la utilidad de esos documentos, que guían y orientan en el descubrimiento del territorio y de su historia. Se presta gran atención a los lugares y a las instalaciones relacionadas con los oficios y las actividades tradicionales (por ejemplo, granjas, empresas jesuitas, fábricas, vías y estructuras relacionadas con las actividades de trashumancia) y a la historia temprana (sitios arqueológicos).

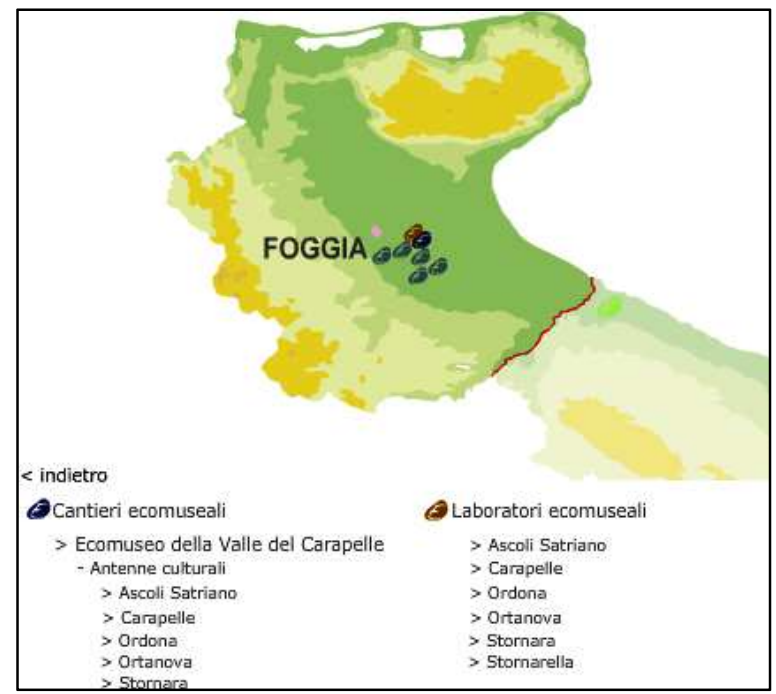

Figura 6 - Ecomuseo della Valle del Carapelle Fuente: Sistema Ecomuseale della Puglia.

\section{Del Ecomuseo al Mapa de Comunidad: técnica participativa para un desarrollo sustentable}

Los casos presentados se caracterizan por tener las mismas pautas de acción en lo referente a la creación de los mapas de comunidad, tal como indicado por A. Summa, que las reúne en ocho puntos. 
1. Presentación de la iniciativa "Ecomuseo": durante esa fase de sensibilización de administración y ciudadanos, se organizan reuniones para permitir la libre circulación de contenidos y objetivos del proyecto, recoger sugerencias, adhesiones y reacciones. Es una ocasión para explicar la importancia de la participación del mayor número de habitantes. La presentación a las principales instituciones está finalizada a la obtención de su aprobación y cooperación. Para comunicar el lanzamiento del proyecto a todos los ciudadanos, se distribuyen invitaciones y se publican carteles; el lenguaje gráfico- visual del material promocional debe ser fácilmente comprensible. También se envían notas de prensa. Durante la presentación se explican a los habitantes las motivaciones, se aclara el proceso de elaboración del instrumento principal de la lectura participativa del territorio: el mapa de Comunidad. El proyecto puede atraer de inmediato la atención de un primer núcleo de personas que ofrece su disponibilidad en contribuir al éxito de la idea.

2. Fase de observación directa de las características espaciales-territoriales: el grupo de participantes más significativo está formado por los componentes más adultos (mejores conocedores del territorio). Este colectivo posee una experiencia más profunda del territorio, por lo que puede generar curiosidad. A través de un lenguaje sencillo, intercalado con proverbios, refranes, canciones y poemas, los miembros más antiguos del grupo proporcionan una cantidad considerable de información útil para reconstruir la red de relaciones entre fenómenos físicos y los provocados por el hombre. El objetivo del grupo de trabajo no es sólo observar los aspectos físicos que caracterizan el paisaje, sino ser capaz de poner de relieve la relación entre el hombre (y sus necesidades) y la naturaleza (con sus recursos y leyes) con el fin de entender los cambios. Se inicia, por lo tanto, con la discusión y la observación directa de esas características espaciales.

3. Fase de análisis de la presencia humana: es un proceso largo y meticuloso, siempre basado en el diálogo continuo, sin limitar el flujo de la historia. Tras haber consensuado los argumentos, cada miembro del grupo puede contribuir en la construcción de Mapa de la Comunidad. Para esto es importante entender los intereses e inclinaciones individuales, para repartir tareas específicas.

4. Reuniones de grupos de trabajo: tienen lugar con cadencia quincenal. Los grupos de trabajo deben reflejar la diversidad de las distintas actividades; entre sus tareas está la 
recogida de material e información inferidos a partir de comparaciones con otras personas que no toman parte activa en las reuniones del Laboratorio.

5. Fase de recogida de informaciones: en esa fase pueden darse problemas durante la realización de cuestionarios debido a la desconfianza hacia prácticas participativas, aunque ese solo interese cuestiones relativas al conocimiento del territorio. Por ello, en muchos casos se cuenta con la colaboración de las escuelas. Otra técnica son las reuniones formales e informales, que permiten recoger informaciones de forma directa.

6. Fase de definición del marco territorial: los grupos de trabajo establecen el contexto territorial de referencia, identifican los temas a analizar y realizan una contribución de ideas y direcciones. En general, es adecuado basar la estrategia de detección en dos actividades principales: 1) Investigación documental y de archivo como objetivo para formar una primera base de conocimientos (donde las fuentes consultadas fueron bibliográfica, oral, archivo y fotográfica); 2) Verificación de la información obtenida a través de una serie de conversaciones y entrevistas con los depositarios del conocimiento y la detección en el campo de los métodos y procedimientos de conocimiento local. Especial atención se dirige tanto a los resultados que al proceso, con la experimentación de diferentes metodologías, cuales la escucha, la selección/decisión de los elementos y valores formalmente representados en el mapa. Para sistematizar la cantidad de información se fijan unas categorías temáticas: 1 . Patrimonio construido; 2 . Herencia agro-silvicultura y pastoreo; 3. Costumbres y tradiciones; 4. Patrimonio arqueológico; 5. El conocimiento y locales producciones. Cada área cuenta con un referente, que se compromete en la gestión de datos y material iconográfico relacionado el tema. Tras reuniones y reflexiones, todos los participantes del grupo deciden los elementos que merecen ser representados en los mapas de comunidad.

7. Diseño del mapa: se realiza en forma de mapa cartográfico o por cualquier otro medio de representación, producidos y elaborados para que la población pueda reconocerse. Así que, a partir de la cartografía tradicional se insertan los elementos territoriales destacados. Se procede a la fusión con los diseños del grupo, que deben de ser comprensibles por parte de los habitantes. Para ello, se pueden introducir explicaciones.

8. Exhibición del mapa: es una fase de condivisión del trabajo. 
9. Realización del cuaderno: se describe el proceso de participación y realización del mapa, los temas tratados y las experiencias.

Los resultados obtenidos a partir de la elaboración de los mapas permiten poner en marcha la segunda fase de la construcción pública del paisaje. Desde el punto de vista de la gestión de la administración, los mapas de comunidad representan un proyecto consensuado del paisaje. A través de esta planificación compartida y consciente, se fomenta una actitud activa y responsable hacia el territorio (CARTA, 2011). La administración se acerca a la población, descubriendo sus saberes y encontrando respuestas útiles para aclarar aspectos relacionados con la riqueza del patrimonio y la participación de la comunidad local, con la transmisión de conocimientos y la comunicación de la investigación. Se podría considerar una nueva "forma de diálogo y comunicación escucha" de la ciudadanía, que puede obviar a problemas. Para la comunidad local, el mapa es un camino colectivo basado en la participación, la investigación y el compromiso; a través del mismo se fortalece el vínculo entre gente y lugares (BARATTI, 2012). Es una herramienta de valorización del territorio, en cuanto permite reflexionar sobre la calidad de los sitios, su potencial y significados.

\section{Análisis de las potencialidades de los Ecomuseos para un desarrollo rural sostenible}

Las experiencias anteriores demuestran de qué manera el ecomuseo promueve dinámicas sociales positivas que mejoran la calidad de vida de los ciudadanos, beneficiados por la institución del ecomuseo. El planteamiento participativo que resulta de los mapas de comunidad puede ser considerado una oportunidad para los ciudadanos de involucrarse y de representar el patrimonio, el paisaje y en general su saber territorial, que ha quedado plasmado y compartido. Se trata de una participación que queda plasmada en la externalización de imágenes del territorio, que se sustenta en atributos, recuerdos, transformaciones, realidad actual y visiones deseadas de futuro. Por todo ello, se podría abordar un nuevo concepto de territorio que reúne la historia de sus habitantes, una 
herencia material e inmaterial. En el caso de Apulia, los mapas de comunidad se han convertido en recursos útiles para la redacción del PPTR, como demuestra su utilidad para la "concienciación del lugar" a través de la participación ciudadana en la construcción de valores patrimoniales, territoriales y paisajísticos.

Otras de las potencialidades derivadas de los ecomuseos conciernen su factor innovador para los nuevos escenarios económicos, puesto que pueden facilitar el desarrollo local. El análisis pretende contestar a una pregunta clave: ¿Cómo contribuyen los ecomuseos a trasformar y mejorar realidades territoriales en crisis en espacios emergentes rurales innovadores?

- Crean oportunidades de empleo para las poblaciones locales, en cuanto suponen la puesta en marcha de proyectos que requieren el esfuerzo de la población.

- Fomentan el desarrollo económico endógeno redescubriendo el valor de recursos medioambientales y patrimoniales poco valorados o en riesgo de abandono. Además permiten la recuperación de actividades vinculadas a la artesanía y al sistema agroalimentario tradicional.

- La actividad de las asociaciones es fundamental para reconstruir tradiciones, costumbres y modos de vida y para dar voces al pasado. El factor social que las sostiene es la clave de su éxito.

- El redescubrimiento del legado material e inmaterial del ámbito territorial de referencia se convierte en recurso didáctico, que permite sensibilizar a la ciudadanía activando una red de iniciativas locales. Se ponen en marcha nuevas formas de pensar el territorio.

- El redescubrimiento del legado material e inmaterial del espacio del ecomuseo construye un sistema favorable al aprovechamiento turístico, preparando itinerarios, rutas, visitas y ofreciendo servicios.

- En el marco de la recuperación de valores y costumbres del pasado, promueve estructuras residenciales e históricas de valor artístico que embellecen el espacio museal.

- Los ecomuseos permiten acondicionar a un espacio con múltiples valores y funcionalidades (didácticos, informativos, con centros de interpretación, de documentación e información, centros de información turística, etc.). 
Desde luego, una correcta planificación requiere también visiones de futuro a medio plazo. Por lo tanto, para evitar que esos ecomuseos agoten las energías de sus impulsores y que queden como contenedores vacíos será necesario seguir contando con la participación ciudadana y la voluntad política de mantener vivo el proyecto. Para ello, se aconsejan:

1. Estudio de la comunidad: para monitorear sus puntos fuertes y débiles, las posibles organizaciones y fundaciones colaboradoras, así como la implicación que se puede esperar de los ciudadanos en términos globales.

2. Estudios de sostenibilidad de los ecomuseos: para fijar prioridades y líneas futuras de intervención. En esta planificación habrá que tener en cuenta a las nuevas generaciones, su sensibilidad y su interés hacia el territorio, dos elementos claramente distintos entre generaciones.

Estos estudios son necesarios si se postula que los ecomuseos son entidades vivas, cambiantes y sin un modelo único y rígido (RIVIÈRE, 1985).

\section{Conclusiones}

En una realidad alterada por los cambios de la situación económica, en este artículo se ha querido poner de manifiesto el hecho de que el ecomuseo permite la creación de nuevos espacios dotados de herramientas que facilitan la recuperación y la valorización del territorio. En calidad de museos permanentes, los ecomuseos pueden volverse lugares activos en los cuales promover la identidad colectiva y el patrimonio cultural, medioambiental y paisajístico. Las experiencias presentadas están acomunadas por el deseo de poner en valor los recursos territoriales a través de una nueva imagen que resulte de la interacción de factores geográficos, paisajísticos y medio-ambientales. En estos casos, los ecomuseos han contribuido a elaborar nuevos modelos de desarrollo local, además de recuperar e reinterpretar significados y valores territoriales y paisajísticos. Desde un punto de vista económico, esta metodología se ha fundado en la valorización de recursos endógenos materiales e inmateriales (tradiciones, historia, recursos humanos, etc.), junto a 
la participación ciudadana. Además, en un contexto de crisis, la valorización del territorio por parte de los ciudadanos les ofrece una posibilidad de empleo.

El avance de los ecomuseos en Apulia en los últimos años ha fomentado el diseño de nuevos herramientas y métodos de conocimiento y la mejora del paisaje en relación al desarrollo local y auto-sostenible. El proyecto experimental de los mapas de comunidad ha sido una buena práctica, con efectos positivos en la redacción del Plan Paisajístico, primer Plan regional que experimenta formas activas de participación en aplicación del Convenio Europeo del Paisaje.

Por lo que se refiere a los Mapas de las Comunidades, esos elementos de planificación y gestión territorial han permitido y seguirán permitiendo a los habitantes participar en la representación del patrimonio, del paisaje y del conocimiento. Las experiencias citadas confirman que la comunidad local puede convertirse en la principal protagonista de este proceso participativo, ya que a través del mapeo comparte su visión del territorio, su percepción, y, de este modo, añade valor a su propio territorio, sus recuerdos, sus transformaciones a su realidad actual y en lo que ella querría que se convirtiera en el futuro. El proceso de construcción del mapa de la comunidad permite a todos los que son interesados observar y escuchar, para descubrir la existencia de diferentes perspectivas desde las cuales ver un ambiente que era familiar, y que se cree ya ampliamente conocido.

En conclusión, a partir de las actividades y de los objetivos planteados los ecomuseos pueden ser definidos "espacios histórico-didácticos" que guardan el pasado de un territorio a la vez que lo enseñan. Estas fórmulas proyectuales del territorio ayudan a leerlo, interpretarlo y darle valor, ofreciendo soluciones de cara situaciones de crisis, puesto que se basan en recursos endógenos.

\section{Bibliografía}

BOLLETTINO UFFICIALE DELLA REGIONE PUGLIA - n. 108 del 08-07-2011. Legge regionale 6 luglio 2011, n. 15 "Istituzione degli ecomusei della Puglia".

BARATTI, Francesco. Ecomusei, Paesaggi e Comunità. Esperienze, progetti e ricerche nel Salento. Milán: Franco Angeli, 2012. ISBN: 9788820410247. 
BELTRÁN GIL, ISABEL. EL Papel Del Ecomuseo Como Elemento DINAMIZADOR DEL DESARROLLO RURAL. En de Cos Guerra, OLGA, REQUES VELASCO, PEDRO (Eds.): La Población En Clave Territorial: Procesos, Estructuras $Y$ Perspectivas $Y$ Análisis. Actas Xiii Congreso De La Población Española, MINISTERIO DE ECONOMÍA Y COMPETITIVIDAD, DIRECCIÓN GENERAL DE INVESTIGACIÓN Y GESTIÓN DEL PLAN NACIONAL DE I+D+I 2012, P. 192-197. ISBN 978-84-695-4480-8

BERGDAHL, EWA. ECOMUSEO DE BERGSLAGEN. Revista De Museología, № o 14, 1998, P. 148-154.

CARTA, Massimo. La rappresentazione nel Progetto del Territorio. Un libro illustrato. Florencia: Florence University Press, 2011. ISBN: 978-88-6453-211-0

COMUNE DI LOCOROTONDO, Ecomuseo della Valle d'Itria: http://www.comune.locorotondo.ba.it/flex/cm/pages/ServeBLOB.php/L/IT/IDPagina/1621

CONSEJO DE EUROPA. Convenio Europeo del Paisaje. Estrasburgo, 2000.

DE VARINE, Hugues. El ecomuseo. Una palabra, dos conceptos, mil prácticas. Mus-A: Revista de los museos de Andalucía, no 8, 2007, p. 19-29.

ECOMUSEI DI VALLE D'ITRIA: http://www.ecomuseovalleditria.it/che-cos-e-l-ecomuseo/la-storia.htm

FERNÁNDEZ RODRÍGUEZ, Carlos. El Ecomuseo De Sierra Mágina: Una Propuesta De Desarrollo Local a Través Del Patrimonio. Sumuntán: anuario de estudios sobre Sierra Mágina, nN20, 2004, p. 105-116.

MORIL, R. Los ecomuseos como instrumento de conservación e interpretación del patrimonio: el museo integral. II Congreso de Verano sobre patrimonio y paisaje en la Sierra de Espadán: Ecomusesos en la Sierra de Espadán, 2009.

MUÑIZ JAÉN, Ignacio. El proyecto municipal del Ecomuseo del Río Caicena (Almedinilla-Córdoba): Patrimonio y desarrollo local desde el mundo rural. E-rph. Revista electrónica de Patrimonio Histórico, no 1, 2007, p. 272. Disponible en: http://dialnet.unirioja.es/servlet/articulo?codigo=4012999

REGIONE PUGLIA. Le mappe di comunità nel piano paesaggistico territoriale della Regione Puglia. (Disponible en: http://paesaggio.regione.puglia.it/images/stories/Mappe COMUNIT/mappe comunita dossier.pdf

REGIONE PUGLIA: Puglia Musei http://pugliamusei.it/tag/regione-puglia/

RIVIÈRE, Georges Henri. Definición evolutiva del ecomuseo. Museum, no 148, Vol XXXVII, n 4, 1985. Páris: Unesco.

SANTACANA MESTRE, Joan y SERRAT ANTOLÍ, Núria: Museografía didáctica. Barcelona: Ariel, 2007. ISBN: 9788434452367

SISTEMA ECOMUSEALE: http://www.ecomuseipuglia.net/home.php

SUMMA, Aldo:_La costruzione di una Mappa di Comunità. En_REGIONE PUGLIA. Le mappe di comunità nel piano paesaggistico territoriale della Regione Puglia. (Disponible en: http://paesaggio.regione.puglia.it/images/stories/Mappe COMUNIT/mappe comunita dossier.pdf) 